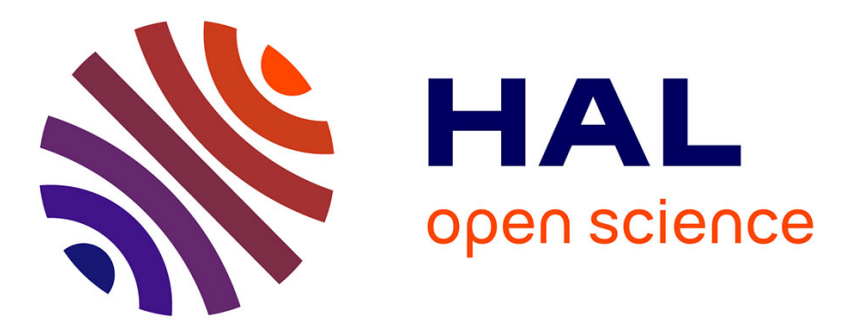

\title{
A Multiscale Approach to Predict Classical Losses in Soft Magnetic Composites
}

Adelina Bordianu, Olivier de La Barrière, Oriano Bottauscio, Alessandra

Manzin, Mario Chiampi

\section{- To cite this version:}

Adelina Bordianu, Olivier de La Barrière, Oriano Bottauscio, Alessandra Manzin, Mario Chiampi. A Multiscale Approach to Predict Classical Losses in Soft Magnetic Composites. IEEE Transactions on Magnetics, 2013, 48 (4), pp. 1537 - 1540. hal-00825526

\section{HAL Id: hal-00825526 \\ https://hal.science/hal-00825526}

Submitted on 24 May 2013

HAL is a multi-disciplinary open access archive for the deposit and dissemination of scientific research documents, whether they are published or not. The documents may come from teaching and research institutions in France or abroad, or from public or private research centers.
L'archive ouverte pluridisciplinaire HAL, est destinée au dépôt et à la diffusion de documents scientifiques de niveau recherche, publiés ou non, émanant des établissements d'enseignement et de recherche français ou étrangers, des laboratoires publics ou privés. 


\section{${ }_{2}^{2} \mathrm{~S}^{\mathrm{o}}$} OFT MagnetiC Composites (SMC) appear as very grains.

in high speed electrical machines [1]. Indeed, their granular structure leads to a significant reduction of the classical loss contribution, which, however, can become important at the frequency increase [2]. Moreover, unlike conventional laminated materials, SMC exhibit isotropic magnetic properties, which can make them suitable in machines with 3D flux paths, such as axial flux machines [3]. They also possess isotropic thermal properties [4], which is an important feature for loss dissipation.

An accurate loss prediction is essential for a reliable optimization of electrical machines using such materials. Since the classical loss gives the most important contribution in high frequency applications, this paper focuses on a modeling approach for the computation of eddy currents in SMC materials.

SMC are composed of grains made of a magnetic material (often pure iron). The grains are resin-insulated from each other and then compressed under high pressure. During the compaction, random contacts can appear between grains, as pointed out in [5]. These random contacts are responsible for a SMC bulk conductivity, which is much lower than the one of the grains, but not negligible, giving rise to eddy current circulation at macroscopic scale (sample size).

A model for computing the SMC equivalent permeability taking into account the grain contacts has been proposed in [6]. Here, a micrograph image is exported to an electromagnetic finite element software and used to deduce an equivalent permeability, which is found in good agreement with the measurements. Such an approach could 2 be extended also to eddy current computations, but it is

\title{
A Multiscale Approach to Predict Classical Losses in Soft Magnetic Composites
}

\author{
Adelina (Samoilescu) Bordianu ${ }^{1}$, Olivier de la Barrière ${ }^{2}$, Oriano Bottauscio ${ }^{2}$, Mario Chiampi ${ }^{3}$, and \\ Alessandra Manzin² \\ ${ }^{1}$ Electrical Engineering Department, University "Politehnica" of Bucharest, Bucharest RO-060042, Romania \\ ${ }^{2}$ Istituto Nazionale di Ricerca Metrologica (INRIM), Torino I-10135, Italy \\ ${ }^{3}$ Dipartimento di Ingegneria Elettrica, Politecnico di Torino, Torino I-10129, Italy
}

This paper presents the application of a finite element multiscale method, based on the homogenization technique, to the prediction of classical losses in soft magnetic composite materials. The experimental results, obtained for a wide range of frequencies and for various toroidal samples with different cross sections, are explained by using the considered model. It has been found that the classical losses are influenced by the dimensions of the sample, as well as by the conductivity and the length of the random contacts between the

\section{Index Terms - Composite materials, numerical analysis, multiscale problem, classical losses.}

Manuscript received August 15, 2011

Corresponding author: Olivier de la Barrière (e-mail: barriere@satie.enscachan.fr).

53 limited to very small samples because of the huge number of 54 mesh elements required for a reliable discretization. Other 55 authors have analyzed so small samples that the effects of 56 current paths flowing from grain to grain at the sample scale 57 are negligible [7]. Only the microscopic eddy currents 58 flowing at the grain scale have been taken into account, 59 considering rectangular grains. However, this simplifying 60 assumption is usually not verified in electrical machine 61 applications, where non negligible intergrain currents are 62 present. Therefore, in [8] the classical loss contribution has 63 been evaluated by introducing a loss decomposition into two 64 terms: the first one is due to the eddy currents at the grain 65 scale, the second one is related to the eddy currents at the 66 macroscopic scale and linked to the bulk resistivity. 67 However, this loss summation is not straightforward from a 68 mathematical point of view.

This paper proposes the use of a method based on a multi70 scale homogenization technique [9], successfully applied to different non-homogeneous materials, such as ferrites [10] The main difficulty encountered here is to model the random contacts between grains using the homogenization method. In the first part of the paper, the experimental evidence of the macroscopic eddy currents is shown. Then, the idealized model of the contacts between grains, to be used in the homogenization process, is introduced. The last part of the paper presents the comparison between the experimental results and the model.

\section{EXPERIMENTS}

The presence of macroscopic eddy currents flowing from grain to grain at the sample scale is put in evidence in this section. This evidence is based on the remark that only the macroscopic eddy current term, unlike the loss contributions at the grain scale (hysteresis, excess, and local eddy currents), can be responsible for a dependence of the specific loss on the sample size and shape. The analysis presented in

Digital Object Identifier inserted by IEEE 
88 [7] shows that a toroid with a square cross section $S=5 \times 5$ $89 \mathrm{~mm}^{2}$ seems to be small enough to neglect macroscopic eddy 90 currents. Indeed, when decreasing the section to $2.5 \times 2.5$ $91 \mathrm{~mm}^{2}$, a negligible change in the specific loss has been 92 observed. Here, the analysis is centered on toroids with cross 93 sections greater than $5 \times 5 \mathrm{~mm}^{2}$, where not negligible 94 macroscopic eddy currents are expected.

\section{A. Description of the experimental set-up}

The considered SMC are produced by the Swedish company Höganäs in bulk cylindrical shape (diameter: 80 $\mathrm{mm}$, height: $20 \mathrm{~mm}$ ), under the reference "Prototyping" [11]. Since these blocks can be quite easily cut, they are well suitable for making toroids of different cross sections, as the ones investigated in this paper and represented in Fig. 1. The first section (toroid A: $S_{\mathrm{A}}=5 \times 5 \mathrm{~mm}^{2}$ ) is considered as the reference case [7], having assumed that the eddy currents are confined at the grain scale. Two larger samples are also considered: toroid $\mathrm{B}\left(S_{\mathrm{B}}=10 \times 10 \mathrm{~mm}^{2}\right)$ and toroid $\mathrm{C}\left(S_{\mathrm{C}}=\right.$ $\left.10 \times 20 \mathrm{~mm}^{2}\right)$. The external diameter $D_{\text {ext }}$ of the toroids is always set at its maximal value (i.e. the external diameter of the blocks, equal to $80 \mathrm{~mm}$ ), in order to ensure a uniform flux density distribution throughout the entire cross section.

It is also important to give some details about the cutting procedure. Indeed, some authors [12] have pointed out the fact that a mechanical abrasion could create conductive paths on the material surface. This could introduce criticalities in our experiments: if the four faces of the toroid are made conductive by the cutting abrasion, a fictitious closed electrical turn would be created on the toroid surface. This is another reason why the toroids have an external diameter equal to the external diameter of the blocs $D_{\text {ext }}=80 \mathrm{~mm}$. This choice ensures that at least the external face has not undergone any abrasion, therefore making impossible the formation of a closed conductive turn around the toroids.

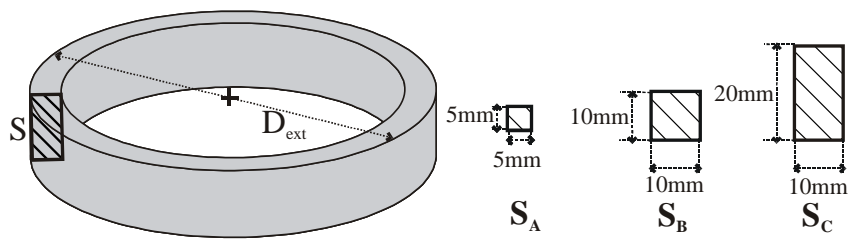

Fig. 1. Scheme of the toroidal sample and of the different cross sections considered in the analysis.

The experimental loss measurement is carried out as follows. The toroid is wound by two windings. The primary winding is supplied under AC conditions with a linear power amplifier. The current flowing in this primary winding is measured using a resistive sensor. This allows to deduce the applied magnetic field, which is, according to Ampere's law, proportional to the current. The mean flux density over the toroid cross section is measured by the integration of the voltage given by a secondary winding. Then, the $B-H$ curve is deduced, and the area of the cycle gives the total specific loss per cycle $W_{t o t}$. A feedback loop permits to get a perfect sinusoidal polarization in the toroid, assumed to be equal to the magnetic flux density (frequency $f$, peak value $J_{p}$ ).

\section{B. Loss results in the different toroids}

The total specific energy loss per cycle $W_{t o t}\left(J_{p}, f\right)$ measured in the considered toroids are given in Fig. 2. The dependence of the specific loss on the toroid cross section due to macroscopic eddy current patterns is well evident.

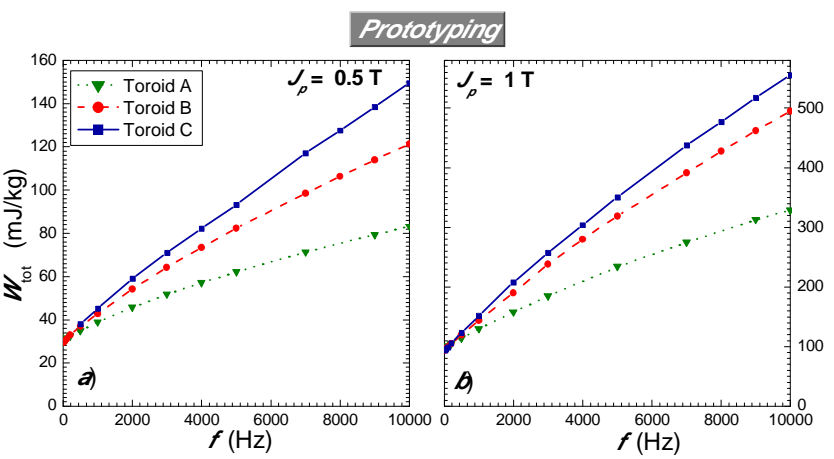

Fig. 2. Total measured loss $W_{\text {tor }}\left(J_{p}, f\right)$ versus frequency $f$ for $J_{p}=0.5 \mathrm{~T}$ (a) and $J_{p}=1 \mathrm{~T}(\mathrm{~b})$

\section{Assumption of no skin effect for the loss separation}

The loss separation has been carried out following the approach described in [7]. Starting from the assumption of only local eddy currents in toroid A, a model of eddy currents at the scale of the single grain has been developed. The hysteresis and excess losses, which are local per definition, have been extracted. Then, we assume that, under the same excitation conditions (frequency $f$ and polarization peak value $J_{p}$ ), the excess and hysteresis losses in toroids B and $\mathrm{C}$ are equal to the ones in toroid $\mathrm{A}$, in spite of the presence of macroscopic eddy currents which change the classical loss. Therefore, the classical loss contribution in toroids $\mathrm{B}$ and $\mathrm{C}$ is computed by subtracting hysteresis and excess loss obtained in toroid A from the total measured loss.

This procedure is valid if we assume that the macroscopic eddy current patterns do not modify the local magnetic flux density in the toroid. In other terms, the skin effect at the sample scale must be negligible. To verify this assumption experimentally the local magnetic flux density in the cross section of toroid $\mathrm{C}$ has been measured, by drilling two holes in the toroid, as represented in Fig. 3. An internal coil between the two holes allows us the measurement of the internal magnetic flux density $B_{\text {int }}(t)$, while an external winding enables the detection of the external field $B_{\text {ext }}(t)$. The skin effect due to macroscopic eddy current patterns is negligible if these internal and external magnetic flux density components do not differ from the mean field $B(t)$ measured at the secondary winding.

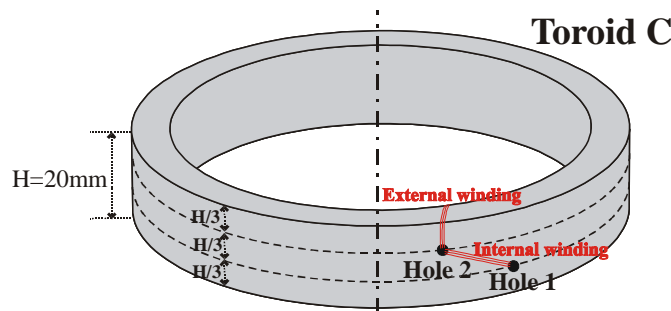

Fig. 3. Scheme of the measurement procedure for the internal and external magnetic flux density components in toroid $\mathrm{C}$. 
The ratios of the peak values of the local and mean magnetic flux densities and the phase shift between their first harmonics are plotted versus frequency for $J_{p}=1 \mathrm{~T}$ in Fig. 4(a) and in Fig. 4(b), respectively. The amplitude of the ratio is close to unity, while the phase shift does not exceed $5^{\circ}$, so that the assumption of negligible skin effect is well justified.

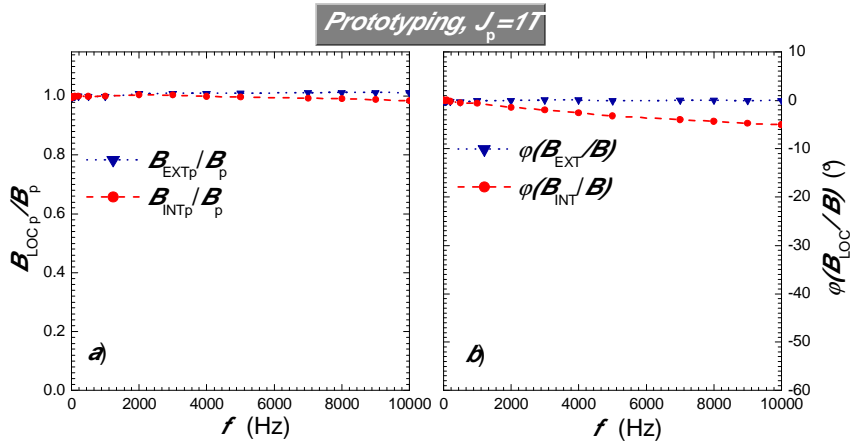

Fig. 4. a) Ratios of the peak values of the local magnetic flux densities, $B_{e x t}(t)$ and $B_{\text {int }}(t)$, and the mean magnetic flux density $B(t)$. b) Phase shifts between the first harmonics of the local and mean magnetic flux densities.

\section{MODELING APPROACH}

The starting point of the modeling approach is the application of the mathematical homogenization technique to infer the physical and geometrical properties of the equivalent contacts between the grains. For such a purpose, the $3 \mathrm{D}$ random distribution of grains, with possible contacts, is replaced with a $2 \mathrm{D}$ periodic structure represented by the repetition of an idealized elementary cell. A known sinusoidal magnetic flux is imposed along the direction normal to this $2 \mathrm{D}$ domain and the electromagnetic field problem is formulated in terms of an electric vector potential with homogeneous Dirichlet boundary conditions [13]. The governing equation is written in the frequency domain expressing the field quantities as phasors. All the field problems involved in the adopted approach are solved through the Finite Element Method.

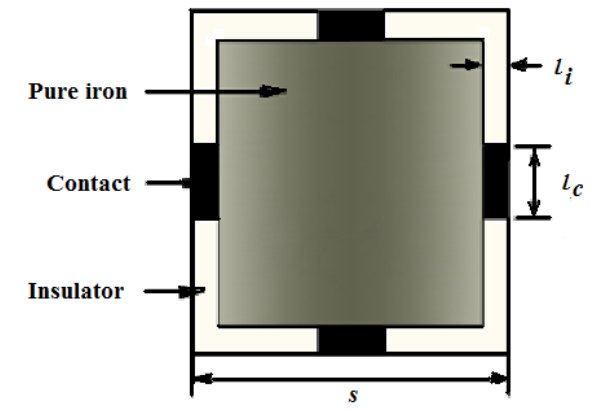

Fig. 5: Scheme of the elementary cell with idealized grain contacts.

For the specific application, the elementary square cell ( $s=$ $115 \mu \mathrm{m})$ shown in Fig. 5 is adopted, where four regions connecting the surrounding cells are introduced to simulate the presence of grain contacts. The idealized square grain is assumed to have the average dimensions of the actual grains, observed in the micrograph images.

Both for grain and contacts the relative magnetic permeability is assumed to be equal to 450 , as deduced by a
3D analysis. However, the precise value of this parameter has a limited impact, due to the absence of skin effect shown in the previous section. The grain is made of pure iron with an electrical conductivity of $10 \mathrm{MS} / \mathrm{m}$. The non-magnetic insulating layer has a thickness $l_{i}=0.74 \mu \mathrm{m}$ and a high resistivity $\left(\rho_{i}=10 \mathrm{M} \Omega \mathrm{m}\right)$. The length and the electrical conductivity of the contact are chosen, by a trial and error procedure, to fit the classical loss contribution. Magnetic fluxes $\Phi$ corresponding to uniform magnetic flux densities of $0.5 \mathrm{~T}$ and $1 \mathrm{~T}$ are imposed along the direction normal to the sample cross-section.

Since the aim is an accurate evaluation of the classical losses at both grain and sample scales, a multiscale model able to reconstruct the macroscopic and local distribution of eddy currents has to be adopted. The mathematical homogenization approach, based on the multiple scale expansion theory [13], is suitable for this application. Indeed, following this technique, the field equation is handled by searching a solution expressed in an asymptotic form, with a zero-order term and higher-order correctors. The first term describes the homogenized solution computed in a homogenous medium with "average" properties; the other terms introduce corrections to reproduce the local behavior of the heterogeneous material. A detailed description of the mathematical formulation can be found in [9]. As an example, the effect of the superimposition of the local terms to the global one is shown in the eddy current distribution presented in Fig. 6.

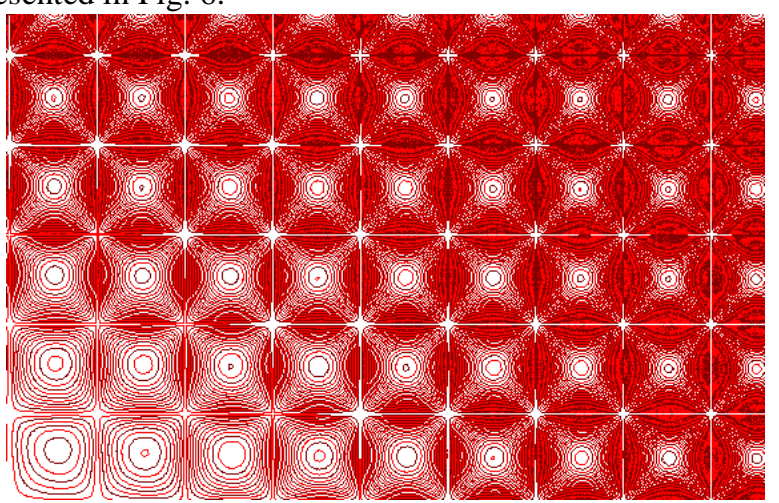

Fig.6. Distribution of current field lines in the cross section of a SMC toroid, computed using the homogenization method with second order correctors.

\section{RESUlt DisCUSSION}

The first problem for the model development is the characterization of the contact, starting from the experimental data. Having fixed the insulating layer thickness, two parameters have to be identified: the contact length $l_{c}$ and its conductivity $\sigma_{c}$. To investigate the influence of these parameters on the classical loss component, $l_{c}$ is varied from $\sim 0.025 \mu \mathrm{m}$ up to $115 \mu \mathrm{m}$ (full contact) and $\sigma_{c}$ is varied in the range between $\sim 20 \mathrm{~S} / \mathrm{m}$ and $\sim 100000 \mathrm{~S} / \mathrm{m}$.

A first analysis, performed keeping constant one of the two parameters, shows that the increase of the other one always leads to an increase in the macroscopic eddy currents and in the classical loss too. However, when the two 
257 parameters are simultaneously modified maintaining their 258 product constant, the classical loss remains practically 259 unchanged in the whole range of contact length. This implies 260 that a single parameter (the product $\sigma_{c} l_{c}$ ) can fully indentify 261 the properties of the contact.

262 The identification process is performed considering the 263 total loss measured in toroid B at $7000 \mathrm{~Hz}$ from which the 264 hysteresis and excess losses obtained in toroid $\mathrm{A}$ are 265 subtracted to evaluate the classical loss contribution. A value 266 of $2850 \mathrm{~S}$ has been determined for the product $\sigma_{c} l_{c}$ and 267 adopted in all the simulations carried out in the frequency 268 range from $1 \mathrm{kHz}$ to $10 \mathrm{kHz}$, where the SMC components 269 usually operate and the influence of eddy currents is better 270 highlighted.

271 The comparison between the experimental and computed 272 classical losses in toroids B and C are presented in Fig. 7 for 273 two flux values, corresponding respectively to uniform flux 274 densities of $0.5 \mathrm{~T}$ and $1.0 \mathrm{~T}$.

275
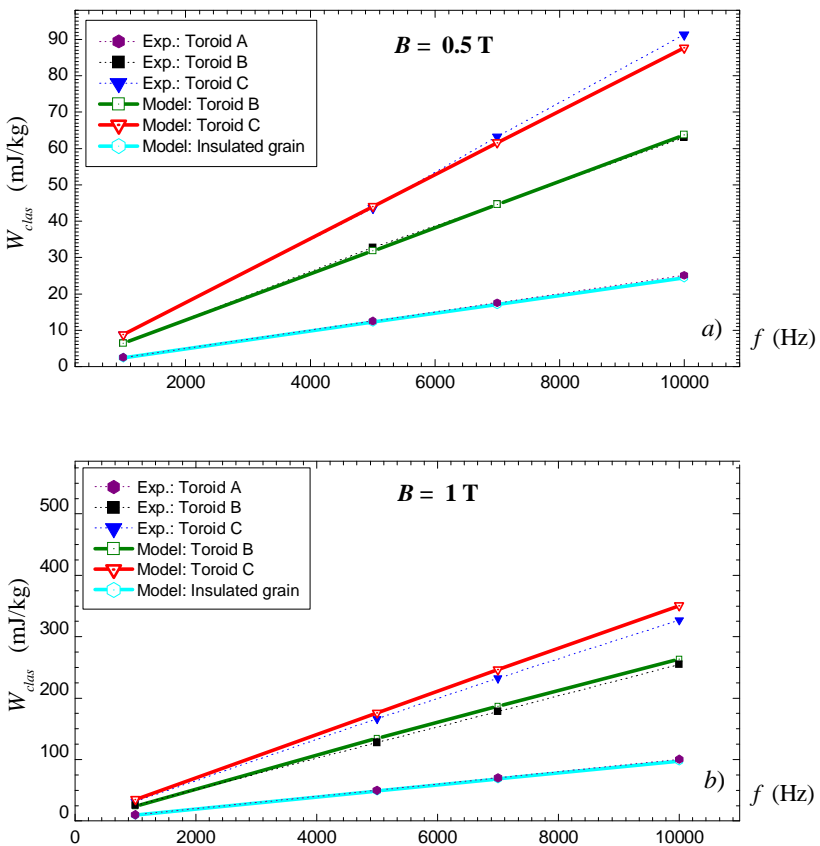

Fig. 7. Classical losses $W_{\text {clas }}$ as a function of frequency $f$, for a magnetic flux density of $0.5 \mathrm{~T}$ (a) and $1 \mathrm{~T}(\mathrm{~b})$

For the both toroids the model provides results in very good agreement with the experiments. The relative error is less than $3 \%$ for a magnetic flux density of $0.5 \mathrm{~T}$ and it is approximately $6 \%$ for $1 \mathrm{~T}$. The errors can be attributed both to the measurement uncertainty and to the approximations intrinsic in a numerical model. The greater errors in the $1 \mathrm{~T}$ case can be explained by the higher temperature increase in the sample, consequent to the loss enhancement, which can modify the material properties. For both sections, the influence of macroscopic eddy currents cannot be disregarded and it is responsible of the dependence of the classical losses on the sample size. For toroid A, a good agreement with the numerical model is also found when simulating insulated grains (i.e. the circulation of macroscopic grains is neglected).

\section{CONCLUSION}

The paper proposes a computational method, based on a multi-scale homogenization technique, for the evaluation of the classical loss component in composite materials. The model takes into account the presence of macroscopic eddy currents, which can flow between grains due to random contacts. The comparison between the experimental and numerical results shows a good agreement for toroids with large cross sections. It has been observed that at the increase of the toroid size the current path, which is mainly local for small cross sections, tends towards a more global distribution.

The proposed model is able to evaluate the increase of the classical loss due to the presence of currents at sample scale for any kind of SMC materials, provided that some measurements are available for the material identification.

\section{ACKNOWLEDGMENT}

Mrs. Adelina (Samoilescu) Bordianu work has been funded by the Sectoral Operational Programme Human Resources Development 2007-2013 of the Romanian Ministry of Labour, Family and Social Protection through the Financial Agreement POSDRU/88/1.5/S/60203.

\section{REFERENCES}

[1] A. Chebak, P. Viarouge, and J. Cros, "Analytical Computation of the Full Load Magnetic Losses in the Soft Magnetic Composite Stator of High-Speed Slotless Permanent Magnet Machines," IEEE Trans. Magn., vol. 45, no. 3, pp. 952-955, 2009.

[2] J. Wang, T. Ibrahim, and D. Howe, "Prediction and Measurement of Iron Loss in a Short-Stroke, Single-Phase, Tubular Permanent Magnet Machine," IEEE Trans. Magn., vol. 46, no. 6, pp. 1315-1318, 2010.

[3] G.S. Liew, N. Ertugrul, W.L. Soong, and D.B. Gehlert, "Analysis and Performance Evaluation of an Axial-Field Brushless PM Machine Utilising Soft Magnetic Composites," in IEEE Int. Conf. on Electric Machines \& Drives, Antalya, 2007, pp. 153-158.

[4] F. Marignetti and V.D. Colli, "Thermal Analysis of an Axial Flux Permanent-Magnet Synchronous Machine," IEEE Trans. Magn., vol. 45, no. 7, pp. 2970-2975, 2009.

[5] C. Yanhong and GB. Kliman, "Modeling of soft magnetic composites," in IEEE Industry Applications Conf. (IAS), 2004.

[6] C Cyr, P. Viarouge, S. Clénet, and J. Cros, "Methodology to Study the Influence of the Microscopic Structure of Soft Magnetic Composites on Their Global Magnetization Curve," IEEE Trans. Magn., vol. 45, no. 3, pp. 1178-1181, 2009.

[7] O. de la Barrière et al., "Loss separation in soft magnetic composites," J. Appl. Phys., vol. 109, p. 07A317, 2011.

[8] D. Zidaric, B. Zagirnyak, and M. Miljavec, "Soft magnetic composite in design of BLDC motor," in Int. Conf. on Electrical Machines, Cracow, 2004.

[9] O. Bottauscio, A. Manzin, V.C. Piat, M. Codegone, and M. Chiampi, "Electromagnetic phenomena in heterogeneous media: Effective properties and local behavior," J. Appl. Phys., vol. 100, p. 044902, 2006.

[10] F. Fiorillo, C. Beatrice, O. Bottauscio, A. Manzin, and M. Chiampi, "Approach to magnetic losses and their frequency dependence in MnZn ferrites," Appl. Phys. Let., vol. 89, no. 12, pp. 122513.1-3, 2006.

[11] Höganäs SMC Brochures. [Online]. http://www.hoganas.com/en/Products--Applications/Soft-MagneticComposites/SMC-Brochures-Pics/

[12] C. Cyr, P. Viarouge, J. Cros, S. Clénet, "Resistivity measurement on soft magnetic composite materials," Przeglad Elektrotechniczny, vol. 83, no. 4, pp. 103-104, 2007.

[13] O. Bottauscio, M. Chiampi, and A. Manzin, "Determination of the electromagnetic properties in magnetic composite materials by inverse homogenisation," J. Magn. Magn. Mat., vol. 320, pp. e547e550, 2008. 